\title{
MEASURE-MULTIPLICITY OF THE LAPLACIAN MASA
}

\author{
KEN DYKEMA* \\ Department of Mathematics, Texas A\&M University, College Station, TX 77843-3368, USA \\ e-mail:kdykema@math.tamu.edu \\ and KUNAL MUKHERJEE \\ Institute of Mathematical Sciences, C.I.T Campus, Taramani, Chennai 600113, Tamil Nadu, India \\ e-mail:kunal@imsc.res.in
}

\begin{abstract}
(Received 8 January 2012; revised 18 February 2012; accepted 8 March 2012;
\end{abstract} first published online 2 August 2012)

\begin{abstract}
It is shown that for the Laplacian masa in the free group factors, the orthocomplement of the associated Jones' projection is an infinite direct sum of coarse bimodules.
\end{abstract}

2000 Mathematics Subject Classification. 46L10.

1. Introduction. Let $2 \leq N<\infty$ and consider the free group factor $L\left(\mathbb{F}_{N}\right)$, which is the group von Neumann algebra of the non-abelian free group $\mathbb{F}_{N}$ on $N$ generators, and is a type $\mathrm{II}_{1}$-factor. We denote by $a_{i},(1 \leq i \leq N)$ the canonical unitary generators of $L\left(\mathbb{F}_{N}\right)$ corresponding to the free generators of a free group. A maximal abelian subalgebra (or masa) of a $\mathrm{II}_{1}$-factor is a self-adjoint, commutative subalgebra that is maximal with respect to these properties. Various kinds of masas were introduced by Dixmier [4] and have been extensively studied. See, for example [18]. A generator masa in $L\left(\mathbb{F}_{N}\right)$ is a subalgebra generated by one of the $a_{i}$. There are $N$ of them, and these are easily seen to be singular (see [4] for the definition), as the cyclic subgroup generated by the corresponding group element is malnormal in $\mathbb{F}_{N}$ (see [6]). In fact, more is true. The Pukánszky invariant (see [13]) is $\{\infty\}$ [17], the malnormality above forces the single generator masas to be strongly mixing [6] and the orthocomplement of the associated Jones' projection is a coarse bimodule over the masa [1, Corollary 4.6]. These masas are outer conjugate, and Popa had shown in [11, Corollary 4.3] that these masas are not inner conjugate.

The radial or the Laplacian masa in $L\left(\mathbb{F}_{N}\right)$ is the von Neumann subalgebra generated by $\sum_{i=1}^{N}\left(a_{i}+a_{i}^{-1}\right)$. This subalgebra has been studied extensively for computing the spectra of convolutors and for understanding the harmonic analysis and the representation theory of $\mathbb{F}_{N}$. That this subalgebra is a masa was initially proved by Pytlik [14]. See [16] for an operator-algebraic proof. That the radial masa is singular was proved by Rădulescu [15], and interestingly, the singularity was proved by the calculation of it's Pukánszky invariant, which is also $\{\infty\}$. Several attempts have been made to decide the conjugacy (by an automorphism) of the radial and the single generator masas. However, all conjugacy invariants that have been computed for both the radial and the single generator masas have agreed. For example, they have the

$\overline{{ }^{*} \text { Research }}$ supported in part by NSF grant DMS-0901220. 
same Pukánszky invariant, each is maximal injective $[2, \mathbf{1 2}]$ and each is strongly mixing $[\mathbf{1}$, an argument analogous to Theorem 4.1], [6, 16]. It is known to experts that the single generator and the radial masas are not inner conjugate. However, the problem of deciding the conjugacy as stated above remains open. It is worth noting that there is no natural candidate of a 'radial masa' in $L\left(\mathbb{F}_{\infty}\right)$.

The measure-multiplicity invariant of a masa $A \subseteq M$ was introduced in [10] and named in [5], though in essence it has been known for a long time; it has been studied recently in $[\mathbf{8}, \mathbf{9}]$. It is a way of describing the $A, A$-bimoduled decomposition of $L^{2}(M) \ominus L^{2}(A)$. If the masa $A$ has a separable, unital and weakly dense $C^{*}$ subalgebra isomorphic to $C(Y)$, then the measure-multiplicity invariant of $A$ consists (up to equivalence) of a pair $(\mu, m)$, where $\mu$ is a measure on $Y \times Y$ (which is zero on the diagonal) and a measurable function $m: Y \times Y \rightarrow\{1,2, \ldots, \infty\}$, whose essential range equals the Pukánszky invariant. We call $\mu$ the left-right measure of $A \subseteq M$, and $m$ the multiplicity function. In this paper we calculate the left-right measure of the Laplacian masa $A \subseteq M=L\left(\mathbb{F}_{N}\right)$ and show that it is Radon-Nikodym equivalent to product measure $\lambda \otimes \lambda$, where $\lambda$ is a measure on $Y$ arising from the trace of $M$ restricted to $A$. This shows that the $A, A$-bimodule $L^{2}(M) \ominus L^{2}(A)$ is isomorphic to the direct sum of infinitely many copies of the coarse bimodule $L^{2}(A) \otimes$ $L^{2}(A)$.

That the left-right measure of the Laplacian masa is absolutely continuous with respect to the product measure follows (but not obviously) from results in [16]. However, had the left-right measure been absolutely continuous with respect to the product measure and not equivalent to it, the conjugacy of the single generator and the Laplacian masas would have been settled upon the consideration of the measuremultiplicity invariant and would have implied that the Laplacian masa has no free complement [5]. The left-right measure of every masa in the free group factors must contain a portion of the product measure as a summand. This is a deep theorem of Voiculescu [19]. However, for all known examples of masas in free group factors, the associated bimodule contains a copy of the coarse bimodule as a direct summand. It is worth noting that there are no known examples of masas in $\mathrm{II}_{1}$-factors for which the left-right measure is absolutely continuous with respect to product class and not equivalent to it; a problem that has connections to open questions in spectral realisations of dynamical systems as well; see [1, 9]. Our proof that the left-right measure of the Laplacian masa is indeed the class of product measure relies on Rădulescu's [15] calculations and results.

In Section 2 we recall notations and formulae from [15]. In Section 3 we will compute the left-right measure.

2. Rădulescu's notation and formulae. Here are some notations and facts from [15] that will be used in Section 3. The GNS Hilbert space of $L\left(\mathbb{F}_{N}\right)$ is associated to its trace $\tau$ is $\ell^{2}\left(\mathbb{F}_{N}\right)$. The associated inner product is denoted by $\langle\cdot, \cdot\rangle_{2}$. Let $\mathbb{C}\left[\mathbb{F}_{N}\right]$ denote the group algebra of $\mathbb{F}_{N}$, i.e. the collection of finite sums of the form $\sum_{w \in \mathbb{F}_{N}} \lambda_{w} w, \lambda_{w} \in \mathbb{C}$ equipped with usual product structure. The void word $\emptyset$ corresponds to the identity of $L\left(\mathbb{F}_{N}\right)$. Let $|\cdot|$ denote the word length function on $\mathbb{F}_{n}$. Write

$$
\chi_{n}=\sum_{|w|=n} w, n \geq 0
$$


Thus, $\chi_{0}=1, \chi_{1}$ generates the Laplacian masa and the following recurrence relations hold.

$$
\begin{aligned}
& \chi_{1} \chi_{1}=\chi_{2}+2 N \\
& \chi_{1} \chi_{n}=\chi_{n} \chi_{1}=\chi_{n+1}+(2 N-1) \chi_{n-1}, \quad(n \geq 2) .
\end{aligned}
$$

Let $A=W^{*}\left(\sum_{i=1}^{N}\left(a_{i}+a_{i}^{-1}\right)\right) \subset L\left(\mathbb{F}_{N}\right)$ denote the Laplacian masa. Then it follows from equation (2.1) that $A$ is closure in w.o.t. of $\operatorname{span}\left\{\chi_{n}: n \geq 0\right\}$. Moreover, $\left\{\chi_{n}: n \geq\right.$ 0 \} forms a complete set of orthogonal vectors in $L^{2}(A)$. Furthermore, from equation (2.1) it follows that for all $n \geq 0, \chi_{n}$ is a polynomial in $\chi_{1}$.

For any $l \geq 0$, denote by $M_{0}^{l}$ the span of words of length $l$ in $\mathbb{F}_{N}$. Let $q_{l}$ denote the projection of $\ell^{2}\left(\mathbb{F}_{N}\right)$ onto $M_{0}^{l}$, and let $S_{l} \subseteq M_{0}^{l}$ be the subspace spanned by $\left\{q_{l}\left(\chi_{1} w\right), q_{l}\left(w \chi_{1}\right): w \in \mathbb{F}_{N},|w| \leq l-1\right\}$. Thus, $S_{0}=\{0\}$. For a vector $\xi \in M_{0}^{l}, l \geq 1$, and integers $r, s$ write

$$
\xi_{r, s}= \begin{cases}q_{r+s+1}\left(\chi_{r} \xi \chi_{s}\right) & \text { if } r, s \geq 0 \\ 0 & \text { otherwise }\end{cases}
$$

The next statement is a part of [15, Lemmas 2 and 3]. We state it here for convenience.

LeMmA 2.1. Let $\varepsilon, \varepsilon^{\prime} \in\{ \pm 1\}$ and let $\beta, \beta^{\prime} \in M_{0}^{1} \ominus S_{1}$ be such that

$$
\begin{aligned}
& \beta=\sum_{|w|=1} c_{w} w, \quad \text { with } \quad c_{w}=\varepsilon c_{w^{-1}}, \\
& \beta^{\prime}=\sum_{|w|=1} d_{w} w, \quad \text { with } \quad d_{w}=\varepsilon^{\prime} d_{w^{-1}} .
\end{aligned}
$$

Then for any $n, n^{\prime}, m, m^{\prime} \geq 0$,

$$
\left\langle\beta_{n, m}, \beta_{n^{\prime}, m^{\prime}}^{\prime}\right\rangle_{2}=\delta_{\varepsilon, \varepsilon^{\prime}} \delta_{n+m, n^{\prime}+m^{\prime}}(2 N-1)^{n+m}(-\varepsilon(2 N-1))^{-\left|n-n^{\prime}\right|}\left\langle\beta, \beta^{\prime}\right\rangle_{2} .
$$

Moreover,

$$
\begin{aligned}
\chi_{n} \beta \chi_{m}= & \beta_{n, m}-\left(\beta_{n-2, m}+\beta_{n, m-2}+\varepsilon \beta_{n-1, m-1}\right) \\
& +\sum_{k \geq 2}(-\varepsilon)^{k}\left(\varepsilon \beta_{n-k-1, m-k+1}+\varepsilon \beta_{n-k+1, m-k-1}+2 \beta_{n-k, m-k}\right) .
\end{aligned}
$$

LEMMA 2.2. For $\beta$ as in the statement of the previous lemma and $n, m \geq 0$, we have

$$
\left\langle\chi_{n} \beta \chi_{m}, \beta\right\rangle_{2}= \begin{cases}(-\varepsilon)^{\frac{n+m}{2}} \varepsilon\|\beta\|_{2}^{2}, & n+m>2,|n-m|=2 \\ 2(-\varepsilon)^{n}\|\beta\|_{2}^{2}, & n+m>2, n=m \\ -\|\beta\|_{2}^{2}, & n+m=2,(n, m) \neq(1,1) \\ -\varepsilon\|\beta\|_{2}^{2}, & (n, m)=(1,1) \\ \|\beta\|_{2}^{2}, & (n, m)=(0,0) \\ 0, & \text { otherwise. }\end{cases}
$$


Proof. From Lemma 2.1 we have

$$
\begin{aligned}
\left\langle\chi_{n} \beta \chi_{m}, \beta\right\rangle_{2} & =\left\langle\beta_{n, m}, \beta\right\rangle_{2} \\
& -\left(\left\langle\beta_{n-2, m}, \beta\right\rangle_{2}+\left\langle\beta_{n, m-2}, \beta\right\rangle_{2}+\varepsilon\left\langle\beta_{n-1, m-1}, \beta\right\rangle_{2}\right) \\
& +\sum_{k \geq 2}(-\varepsilon)^{k}\left(\begin{array}{c}
\varepsilon\left\langle\beta_{n-k-1, m-k+1}, \beta\right\rangle_{2}+\varepsilon\left\langle\beta_{n-k+1, m-k-1}, \beta\right\rangle_{2} \\
\left.\quad+2\left\langle\beta_{n-k, m-k}, \beta\right\rangle_{2}\right) .
\end{array}\right.
\end{aligned}
$$

Note that by definition $\beta=\beta_{0,0}$. Using Lemma 2.1 again, we see that the only term in the sum (2.5) that can be non-zero is $k=\frac{n+m}{2}$; moreover, expression (2.4) is non-zero only when $n+m=2$ whereas $\left\langle\beta_{n, m}, \beta\right\rangle_{2}$ is non-zero only when $(n, m)=(0,0)$. From these facts, the formula (2.3) follows easily.

3. Left-right measure. Let $M$ be a separable $\mathrm{II}_{1}$-factor equipped with its faithful normal tracial state $\tau$. This trace induces the Hilbert space norm on $M$, given by $\|x\|_{2}=\tau\left(x^{*} x\right)^{\frac{1}{2}}, x \in M$. The Hilbert space completion of $M$ with respect to $\|\cdot\|_{2}$ is denoted by $L^{2}(M)$. The associated inner product on $L^{2}(M)$ will be denoted by $\langle\cdot, \cdot\rangle_{2}$. Let $M$ acts on $L^{2}(M)$ via left multiplication. Let $J$ denote the Tomita's modular operator on $L^{2}(M)$, obtained by extending the densely defined map $J: M \mapsto M$ given by $J x=x^{*}$. Let $A \subset M$ be a masa. Let $e_{A}$ be the Jones projection associated to $A$. Denote $\mathcal{A}=A \vee J A J$. It is known that $e_{A} \in \mathcal{A}$.

Choose a compact Hausdorff space $X$ such that $C(X) \subset A$ is a norm separable unital $C^{*}$-subalgebra which is w.o.t. dense in $A$. The trace $\tau$ restricted to $C(X)$ gives rise to a probability measure $v$ on $X$. We complete $v$ if necessary so that $A$ is isomorphic to $L^{\infty}(X, v)$.

For definitions and details about measure-multiplicity invariant and left-right measure see $[\mathbf{5}, \mathbf{8}]$. For $\zeta \in L^{2}(M)$, let $\kappa_{\zeta}: C(X) \otimes C(X) \mapsto \mathbb{C}$ be the linear functional defined by

$$
\kappa_{\zeta}(a \otimes b)=\langle a \zeta b, \zeta\rangle_{2}, a, b \in C(X)
$$

Then $\kappa_{\zeta}$ induces a unique complex Radon measure $\eta_{\zeta}$ on $X \times X$ given by

$$
\kappa_{\zeta}(a \otimes b)=\int_{X \times X} a(t) b(s) d \eta_{\zeta}(t, s) .
$$

There is a vector $0 \neq \zeta_{0} \in L^{2}(M) \ominus L^{2}(A)$ such that $\left[\eta_{\zeta_{0}}\right]$ is the left-right measure of $A$.

We will use the result of the following elementary calculation, which we show for convenience.

LEMMA 3.1. Let $x$ be a real or complex number of modulus $|x|<1$, let $\theta$, $\phi$ and $r$ be real numbers. Then

$$
\begin{aligned}
\sum_{n=0}^{\infty} x^{n} \sin n \theta \sin (n+r) \phi= & \frac{1}{2}\left(\frac{\cos (r \phi)-x \cos (\theta+(r-1) \phi)}{1-2 x \cos (\theta-\phi)+x^{2}}\right) \\
& -\frac{1}{2}\left(\frac{\cos (r \phi)-x \cos (\theta-(r-1) \phi)}{1-2 x \cos (\theta+\phi)+x^{2}}\right) .
\end{aligned}
$$


Proof. The left-hand-side of (3.2) equals

$$
\begin{aligned}
- & \frac{1}{4} \sum_{n=0}^{\infty} x^{n}\left(e^{i n \theta}-e^{-i n \theta}\right)\left(e^{i(n+r) \phi}-e^{-i(n+r) \phi}\right) \\
& =-\frac{1}{4} \sum_{n=0}^{\infty} x^{n}\left(e^{i n(\theta+\phi)+i r \phi}-e^{-i n(\theta-\phi)+i r \phi}-e^{i n(\theta-\phi)-i r \phi}+e^{-i n(\theta+\phi)-i r \phi}\right) \\
& =-\frac{1}{4}\left(\frac{e^{i r \phi}}{1-x e^{i(\theta+\phi)}}-\frac{e^{i r \phi}}{1-x e^{-i(\theta-\phi)}}-\frac{e^{-i r \phi}}{1-x e^{i(\theta-\phi)}}+\frac{e^{-i r \phi}}{1-x e^{-i(\theta+\phi)}}\right) .
\end{aligned}
$$

Now putting the first and fourth terms, as well as the second and third terms over a common denominator finishes the calculation.

REMARK 3.2. The Laplacian masa is generated by the self-adjoint operator $\chi_{1}$. The computation of the norm of $\chi_{1}$ and the generating series of its moments goes back to Kesten [7]. From this, using the Stieltjes inversion, the distribution measure of $\chi_{1}$ can be found, and one obtains that the spectrum of $\chi_{1}$ is $\sigma_{\chi_{1}}=[-2 \sqrt{2 N-1}, 2 \sqrt{2 N-1}]$ and the distribution is Lebesgue absolutely continuous. Let us write $a_{N}=2 \sqrt{2 N-1}$. So the weakly dense separable $C^{*}$-subalgebra $C^{*}\left(\left\{1, \chi_{1}\right\}\right)$ of the Laplacian masa $A$ is identified with $C\left[-a_{N}, a_{N}\right]$ in such a way that $\chi_{1}$ is identified with the function $t \mapsto t$ on $\left[-a_{N}, a_{N}\right]$. Thus, from the recurrence relations $(2.1), \chi_{n}$ is identified with a polynomial of degree $n$ in $C\left[-a_{N}, a_{N}\right]$. Also, note that $\chi_{n}, n=0,1, \ldots$, is total family of orthogonal vectors (with respect to $\langle\cdot, \cdot\rangle_{2}$ ) in $L^{2}(A)$; thus, $\frac{\chi_{n}}{\left\|\chi_{n}\right\|_{2}}, n=0,1, \ldots$, is an orthonormal basis of $L^{2}(A)$. Finally, the trace $\tau$ restricted to $A$ is identified with a probability measure $\lambda$ on $\left[-a_{N}, a_{N}\right]$ for which $\left(\chi_{n}\right)_{n \geq 0}$ is a family of orthogonal polynomials. Note that $\lambda$ is the Radon-Nikodym equivalent to the Lebesgue measure on $\left[-a_{N}, a_{N}\right]$. It is straightforward to check from the definition that $\left\|\chi_{n}\right\|_{2}=\sqrt{2 N}(2 N-1)^{\frac{n-1}{2}}$ for all $n \geq 1$.

Consider the function $f:\left[-a_{N}, a_{N}\right] \times\left[-a_{N}, a_{N}\right] \rightarrow \mathbb{R}$ defined by equation

$$
\begin{aligned}
f(t, s)= & \left(1+\frac{\chi_{1}(t) \chi_{1}(s)}{\left\|\chi_{1}\right\|_{2}^{4}}-\frac{\chi_{2}(t)+\chi_{2}(s)}{\left\|\chi_{2}\right\|_{2}^{2}}\right) \\
& +\sum_{n=2}^{\infty}\left(2 \frac{\chi_{n}(t) \chi_{n}(s)}{\left\|\chi_{n}\right\|_{2}^{4}}-\frac{\chi_{n-1}(t) \chi_{n+1}(s)+\chi_{n+1}(t) \chi_{n-1}(s)}{\left\|\chi_{n-1}\right\|_{2}^{2}\left\|\chi_{n+1}\right\|_{2}^{2}}\right) .
\end{aligned}
$$

It is possible to give a closed form of $f$ in terms of transcendental functions. However, for our purpose it is more important to know the 'size' of the zero set of $f$.

Proposition 3.3. The function $f$ defined in equation (3.3) is continuous on $\left[-a_{N}, a_{N}\right] \times\left[-a_{N}, a_{N}\right]$ and real analytic in the interior of this set.

Proof. Let $t \in\left[-a_{N}, a_{N}\right]$ and $\theta \in[0, \pi]$. Consider the homeomorphism $t \mapsto \theta$ from $\left[-a_{N}, a_{N}\right]$ onto $[0, \pi]$ such that

$$
\cos \theta=\frac{t}{a_{N}}, \quad \sin \theta=\frac{\sqrt{a_{N}^{2}-t^{2}}}{a_{N}} .
$$


Then from [3, p. 1062] we have, for all $n \geq 1$,

$$
\frac{\chi_{n}(t)}{(2 N-1)^{\frac{n}{2}}}=\frac{2 \cos \theta \sin n \theta}{\sin \theta}-\frac{2 N}{2 N-1} \cdot \frac{\sin (n-1) \theta}{\sin \theta} .
$$

Write $b=2 N-1$ and $d=\frac{2 N-1}{2 N}$. Then

$$
\frac{\chi_{n}(t)}{\left\|\chi_{n}\right\|_{2}^{2}}=b^{-n / 2}\left(\frac{2 d \cos \theta \sin n \theta-\sin (n-1) \theta}{\sin \theta}\right) .
$$

Since $\frac{|\sin n \theta|}{\sin \theta} \leq n$ for all integers $n \geq 1$ and all $\theta \in[0, \pi]$ (which is easily shown by induction on $n$ ), we have $\frac{\left|\chi_{n}(t)\right|}{\left\|\chi_{n}\right\|_{2}^{2}} \leq 3 n b^{-n / 2}$. Thus, the series in equation (3.3) converges absolutely and uniformly for $(s, t)$ in the domain of $f$. This implies continuity of $f$ on its domain. have

For $t, s \in\left[-a_{N}, a_{N}\right]$, taking $\phi \in[0, \pi]$ so that $\cos \phi=\frac{s}{a_{N}}$ and $\sin \phi=\frac{\sqrt{a_{N}^{2}-s^{2}}}{a_{N}}$, we

$$
\begin{aligned}
\frac{\chi_{n}(t) \chi_{n}(s)}{\left\|\chi_{n}\right\|_{2}^{4}}= & b^{-n}\left(\frac{1}{\sin \theta \sin \phi}\right) \\
& \cdot\left(4 d^{2} \cos \theta \cos \phi \sin n \theta \sin n \phi-2 d \cos \theta \sin n \theta \sin (n-1) \phi\right. \\
& -2 d \cos \phi \sin (n-1) \theta \sin n \phi+\sin (n-1) \theta \sin (n-1) \phi),
\end{aligned}
$$

and one can write a similar expression for

$$
\frac{\chi_{n-1}(t) \chi_{n+1}(s)+\chi_{n+1}(t) \chi_{n-1}(s)}{\left\|\chi_{n-1}\right\|_{2}^{2}\left\|\chi_{n+1}\right\|_{2}^{2}} .
$$

Now summing over $n$ and using Lemma 3.1, we obtain a closed form expression for $f(t, s)$ and see that it is a real analytic function of $\theta$ and $\phi$. Since, for $(t, s)$ in the interior of $\left[-a_{N}, a_{N}\right] \times\left[-a_{N}, a_{N}\right], \theta$ and $\phi$ are real analytic functions of $t$ and $s, f(t, s)$ is indeed a real analytic function of $(t, s)$ there.

THEOREM 3.4. The left-right measure of $A=W^{*}\left(\sum_{i=1}^{N}\left(a_{i}+a_{i}^{-1}\right)\right) \subset L\left(\mathbb{F}_{N}\right)$ is the class of product measure.

Proof. Let $\beta \in M_{0}^{1} \ominus S_{1}$ be as in Lemma 2.1 with $\varepsilon=-1$. Let $\gamma=\frac{\beta}{\|\beta\|_{2}}$. Let $p_{\gamma}$ be an orthogonal projection onto the subspace $\overline{\mathcal{A} \gamma}{ }^{\|\cdot\|_{2}}$. We will show below that the measure class of $\eta_{\gamma}$ on $\left[-a_{N}, a_{N}\right] \times\left[-a_{N}, a_{N}\right]$, which is defined in (3.1), is the same as $\lambda \otimes \lambda$, where $\lambda$ is the measure coming from $\tau$ defined in Remark 3.2. This will imply $\lambda \otimes \lambda \ll v$, where $v$ is the left-right measure of $A$. Indeed (see [5, Sections 5 and 6), the range of $p_{\gamma}$ as a $A, A$-sub-bimodule is a direct integral of the Hilbert spaces with respect to the measure $\eta_{\gamma}$ with multiplicity function being constantly one.

By the proof of [15, Theorem 7], the central support in $\mathcal{A}^{\prime}$ of $p_{\gamma}$ is $1-p_{1}$, and $p_{1}$ is equal to the Jones projection $e_{A}$ onto $L^{2}(A)$. Moreover, by Lemma 6 in [15], it follows that $1-p_{1}$ is greater than or equal to the sum of infinitely many projections all equivalent in $\mathcal{A}^{\prime}$ to $p_{\gamma}$. It follows that $1-p_{1}$ is equal to a sum of projections, infinitely many of which are equivalent in $\mathcal{A}^{\prime}$ to $p_{\gamma}$ and all of which are equivalent in $\mathcal{A}^{\prime}$ to subprojections of $p_{\gamma}$. From this, it follows that the left-right measure $v$ of $A$ has the same measure class as $\eta_{\gamma}$ (cf. [5, Proposition 5.8]). 
Now we go about describing $\eta_{\gamma}$. For $a, b \in C\left[-a_{N}, a_{N}\right]$, one has

$$
\begin{aligned}
& a=\sum_{n=0}^{\infty}\left\langle a, \frac{\chi_{n}}{\left\|\chi_{n}\right\|_{2}}\right\rangle_{2} \frac{\chi_{n}}{\left\|\chi_{n}\right\|_{2}}, \\
& b=\sum_{n=0}^{\infty}\left\langle b, \frac{\chi_{n}}{\left\|\chi_{n}\right\|_{2}}\right\rangle_{2} \frac{\chi_{n}}{\left\|\chi_{n}\right\|_{2}},
\end{aligned}
$$

where the series in the above converge in $\|\cdot\|_{2}$. Thus, using Lemma 2.2 it follows that

$$
\begin{aligned}
\langle a \gamma b, \gamma\rangle_{2}= & \sum_{n, m=0}^{\infty}\left\langle a, \chi_{n}\right\rangle_{2}\left\langle b, \chi_{m}\right\rangle_{2} \frac{\left\langle\chi_{n} \beta \chi_{m}, \beta\right\rangle_{2}}{\left\|\chi_{n}\right\|_{2}^{2}\left\|\chi_{m}\right\|_{2}^{2}\|\beta\|_{2}^{2}} \\
= & \tau(a) \tau(b)+\left\langle a, \chi_{1}\right\rangle_{2}\left\langle b, \chi_{1}\right\rangle_{2} \frac{1}{\left\|\chi_{1}\right\|_{2}^{4}}-\left(\tau(a)\left\langle b, \chi_{2}\right\rangle_{2}+\left\langle a, \chi_{2}\right\rangle_{2} \tau(b)\right) \frac{1}{\left\|\chi_{2}\right\|_{2}^{2}} \\
& +2 \sum_{k=2}^{\infty}\left\langle a, \chi_{k}\right\rangle_{2}\left\langle b, \chi_{k}\right\rangle_{2} \frac{1}{\left\|\chi_{k}\right\|_{2}^{4}}-\sum_{k=1}^{\infty}\left(\left\langle a, \chi_{k}\right\rangle_{2}\left\langle b, \chi_{k+2}\right\rangle_{2}\right. \\
& \left.+\left\langle a, \chi_{k+2}\right\rangle_{2}\left\langle b, \chi_{k}\right\rangle_{2}\right) \frac{1}{\left\|\chi_{k}\right\|_{2}^{2}\left\|\chi_{k+2}\right\|_{2}^{2}} .
\end{aligned}
$$

Consequently from equations (3.3) and (3.4) it follows that

$$
\int_{-a_{N}}^{a_{N}} \int_{-a_{N}}^{a_{N}} a(t) b(s) d \eta_{\gamma}(t, s)=\int_{-a_{N}}^{a_{N}} \int_{-a_{N}}^{a_{N}} a(t) b(s) f(t, s) d \lambda(t) d \lambda(s) .
$$

Thus, $\eta_{\gamma} \ll \lambda \otimes \lambda$ with $\frac{d \eta_{\gamma}}{d(\lambda \otimes \lambda)}=f$.

Write $E=\{(t, s): f(t, s)=0\}$. The intersection of $E$ with the open set $\left(-a_{N}, a_{N}\right) \times$ $\left(-a_{N}, a_{N}\right)$ is a zero set of a real analytic function. It is easy to see (based on induction on the number of variables) that the Lebesgue measure of the zero set of a real analytic function in several variables must vanish, unless the function is identically zero. Thus, we have $(\lambda \otimes \lambda)(E)=0$ and $\eta_{\gamma} \sim \lambda \otimes \lambda$.

\section{REFERENCES}

1. J. Cameron, J. Fang and K. Mukherjee, Mixing and weak mixing abelian subalgebras of type $I I_{1}$ factors (2011), preprint.

2. J. Cameron, J. Fang, M. Ravichandran and S. White, The radial masa in a free group factor is maximal injective, J. Lond. Math. Soc. 82(2) (2010), 787-809. 1065.

3. J. M. Cohen, Operator norms on free groups, Boll. Un. Mat. Ital. B 1(6) (1982), 1055

4. J. Dixmier, Sous-anneaux abeliens maximaux dans les facteurs de type fini, Ann. Math. 59(2) (1954), 279-286.

5. K. Dykema, A. M. Sinclair and R. R. Smith, Values of the Pukanszky invariant in free group factors and the hyperfinite factor, J. Funct. Anal. 240 (2006), 373-398.

6. P. Jolissaint and Y. Stalder, Strongly singular masas and mixing actions in finite von Neumann algebras, Ergodic Theory Dyn. Syst. 28 (2008), 1861-1878. $336-354$.

7. H. Kesten, Symmetric random walks on groups, Trans. Amer. Math. Soc. 92 (1959),

8. K. Mukherjee, Masas and bimodule decompositions of $\mathrm{II}_{1}$-factors, Q. J. Math. 62 (2011), 451-486. 
9. K. Mukherjee, Singular masas and measure-multiplicity invariant, Houston J. Math. (to appear 2013; arxiv:1104.3507).

10. S. Neshveyev and E. Størmer, Ergodic theory and maximal abelian subalgebras of the hyperfinite factor, J. Funct. Anal. 195 (2002), 239-261.

11. S. Popa, Orthogonal pairs of $*$-subalgebras in finite von Neumann algebras, J. Operator Theory 9 (1983), 253-268.

12. S. Popa, Maximal injective subalgebras in factors associated with free groups, $A d v$. Math. 50 (1983), 27-48.

13. L. Pukánszky, On maximal abelian subrings of factors of type $\mathrm{II}_{1}$, Canad. J. Math. 12 (1960), 289-296.

14. T. Pytlik, Radial functions on free groups and a decomposition of the regular representation into irreducible components, J. Reine Angew. Math. 326 (1981), 124-135.

15. F. Rădulescu, Singularity of the radial subalgebra of $\mathcal{L}\left(F_{N}\right)$ and the Pukánszky invariant, Pacific J. Math. 151 (1991), 297-306.

16. A. M. Sinclair and R. R. Smith, The Laplacian masa in a free group factor, Trans. Amer. Math. Soc. 355 (2003), 465-475 (electronic).

17. A. M. Sinclair and R. R. Smith, The Pukánszky invariant for masas in group von Neumann factors, Illinois J. Math. 49 (2005), 325-343 (electronic).

18. A. M. Sinclair and R. R. Smith, Finite von Neumann algebras and masas, London Mathematical Society Lecture Note Series, vol. 351 (Cambridge University Press, Cambridge, UK, 2008).

19. D. Voiculescu, The analogues of entropy and Fisher's information measure in free probability theory III: The absence of Cartan subalgebras, Geom. Funct. Anal. 6 (1996), 172199. 